\title{
AN ULTRAVIOLET SPECTRUM OF THE TIDAL DISRUPTION FLARE ASASSN-14li
}

S. Bradley Cenko ${ }^{1,2}$, Antonino Cucchiara ${ }^{1,3}$, Nathaniel Roth $^{4}$, Sylvain Veilleux $^{2,5}$, J. Xavier Prochaska $^{6}$, Lin Yan ${ }^{7}$, James Guillochon $^{8}$, W. Peter Maksym ${ }^{9,10}$, Iair Arcavi ${ }^{11,12}$, Nathaniel R. Butler ${ }^{13}$, Alexei V. FilipPenko ${ }^{14}$, Andrew S. Fruchter ${ }^{3}$, Suvi Gezari ${ }^{5}$, Daniel Kasen ${ }^{4,14,15}$, Andrew J. Levan ${ }^{16}$, Jon M. Miller ${ }^{17}$, Dheeraj R. Pasham ${ }^{1,2}$, Enrico Ramirez-Ruiz ${ }^{6}$, Linda E. Strubbe ${ }^{18}$, Nial R. Tanvir ${ }^{19}$, and Francesco Tombesi ${ }^{5,20}$

${ }^{1}$ Astrophysics Science Division, NASA Goddard Space Flight Center, Mail Code 661, Greenbelt, MD 20771, USA; brad.cenko@ nasa.gov

${ }^{2}$ Joint Space-Science Institute, University of Maryland, College Park, MD 20742, USA

${ }^{3}$ Space Telescope Science Institute, 3700 San Martin Drive, Baltimore, MD 21218, USA

${ }^{4}$ Department of Physics, University of California, Berkeley, CA 94720, USA
Department of Astronomy, University of Maryland, Stadium Drive, College Park, MD 20742-2421, USA

6 Department of Astronomy and Astrophysics, University of California, Santa Cruz, CA 95064, USA

${ }^{7}$ Infrared Processing and Analysis Center, California Institute of Technology, Pasadena, CA 91125, USA

${ }^{8}$ Harvard-Smithsonian Center for Astrophysics, The Institute for Theory and Computation, 60 Garden Street, Cambridge, MA 02138, USA

${ }^{9}$ Harvard-Smithsonian Center for Astrophysics, 60 Garden Street, Cambridge, MA 02138, USA

${ }^{10}$ Department of Physics and Astronomy, University of Alabama, Tuscaloosa, AL 35487, USA

${ }^{11}$ Las Cumbres Observatory Global Telescope, 6740 Cortona Drive, Suite 102, Goleta, CA 93111, USA

${ }^{12}$ Kavli Institute for Theoretical Physics, University of California, Santa Barbara, CA 93106, USA

${ }^{13}$ School of Earth \& Space Exploration, Arizona State University, AZ 85287, USA

${ }^{14}$ Department of Astronomy, University of California, Berkeley, CA 94720-3411, USA

15 Nuclear Science Division, Lawrence Berkeley National Laboratory, Berkeley, CA, 94720, USA

${ }^{16}$ Department of Physics, University of Warwick, Coventry, CV4 7AL, UK

${ }_{17}^{17}$ Department of Astronomy, The University of Michigan, 1085 South University Avenue, Ann Arbor, MI 48103, USA

${ }^{18}$ Department of Physics and Astronomy \& Carl Wieman Science Education Initiative, University of British Columbia, 6224 Agricultural Road, Vancouver, BC V6T 1Z1, Canada

${ }^{19}$ Department of Physics and Astronomy, University of Leicester, Leicester, LE1 7RH, UK

${ }^{20}$ X-ray Astrophysics Laboratory, NASA/Goddard Space Flight Center, Greenbelt, MD 20771, USA Received 2016 January 12; accepted 2016 February 2; published 2016 February 18

\begin{abstract}
We present a Hubble Space Telescope Space Telescope Imaging Spectrograph spectrum of ASASSN-14li, the first rest-frame ultraviolet (UV) spectrum of a tidal disruption flare (TDF). The underlying continuum is well fit by a blackbody with $T_{\mathrm{UV}}=3.5 \times 10^{4} \mathrm{~K}$, an order of magnitude smaller than the temperature inferred from X-ray spectra (and significantly more precise than previous efforts based on optical and near-UV photometry). Superimposed on this blue continuum, we detect three classes of features: narrow absorption from the Milky Way (probably a high-velocity cloud), and narrow absorption and broad $\left(\sim 2000-8000 \mathrm{~km} \mathrm{~s}^{-1}\right)$ emission lines at or near the systemic host velocity. The absorption lines are blueshifted with respect to the emission lines by $\Delta v=-(250-400) \mathrm{km} \mathrm{s}^{-1}$. Due both to this velocity offset and the lack of common low-ionization features (Mg II, $\mathrm{Fe}$ II), we argue these arise from the same absorbing material responsible for the low-velocity outflow discovered at X-ray wavelengths. The broad nuclear emission lines display a remarkable abundance pattern: $\mathrm{N}$ III], $\mathrm{N}$ IV], and He II are quite prominent, while the common quasar emission lines of $\mathrm{C}_{\text {III] }}$ and $\mathrm{Mg}$ II are weak or entirely absent. Detailed modeling of this spectrum will help elucidate fundamental questions regarding the nature of the emission processes at work in TDFs, while future UV spectroscopy of ASASSN-14li would help to confirm (or refute) the previously proposed connection between TDFs and "N-rich" quasars.
\end{abstract}

Key words: accretion, accretion disks - stars: flare - ultraviolet: general

\section{INTRODUCTION}

A star passing close to a supermassive black hole (SMBH; $M$ $\gtrsim 10^{6} M_{\odot}$ ) will be torn apart by tidal forces (Hills 1975). The accretion of the resulting bound stellar debris results in a luminous transient known as a tidal disruption flare (TDF; Rees 1988). Unlike active galactic nuclei (AGN), the accretion resulting from a TDF is deterministic, and the rate that mass first returns to the SMBH is straightforward to derive (Phinney 1989). For some systems the accretion rate is predicted to transition from highly super-Eddington to subEddington on a time scale of months to years (e.g., De Colle et al. 2012). Furthermore, TDFs can serve as "sign-posts," indicating the presence of a SMBH in galaxies that are otherwise not actively accreting.

Thanks in part to the rapid growth in wide-field optical surveys, the TDF discovery rate has experienced a remarkable increase in recent years. ${ }^{21}$ Optical spectra of PS1-10jh, the first TDF with extensive real-time follow-up observations from such surveys, revealed broad (full width at half-maximum intensity $(\mathrm{FWHM}) \approx 9000 \mathrm{~km} \mathrm{~s}^{-1}$ ) He II emission lines, but lacked detectable Balmer H emission (Gezari et al. 2012). The low $\mathrm{H}: \mathrm{He}$ ratio has led to a vigorous debate within the community, with possible explanations including the disruption of a H-poor star (Gezari et al. 2012; Bogdanović et al. 2014; Strubbe \& Murray 2015); optical-depth effects (Gaskell \& Rojas Lobos 2014) in a radially truncated (but H-rich) broadline region (BLR; Guillochon et al. 2014); complex photoionization processes within an (H-rich) envelope surrounding the accretion disk (Roth et al. 2015); and stellar evolution (i.e., $\mathrm{H}$ burning) in the core of a $\gtrsim 1 M_{\odot}$ star (Kochanek 2015). More

\footnotetext{
${ }^{21}$ See, e.g., https://tde.space.
} 


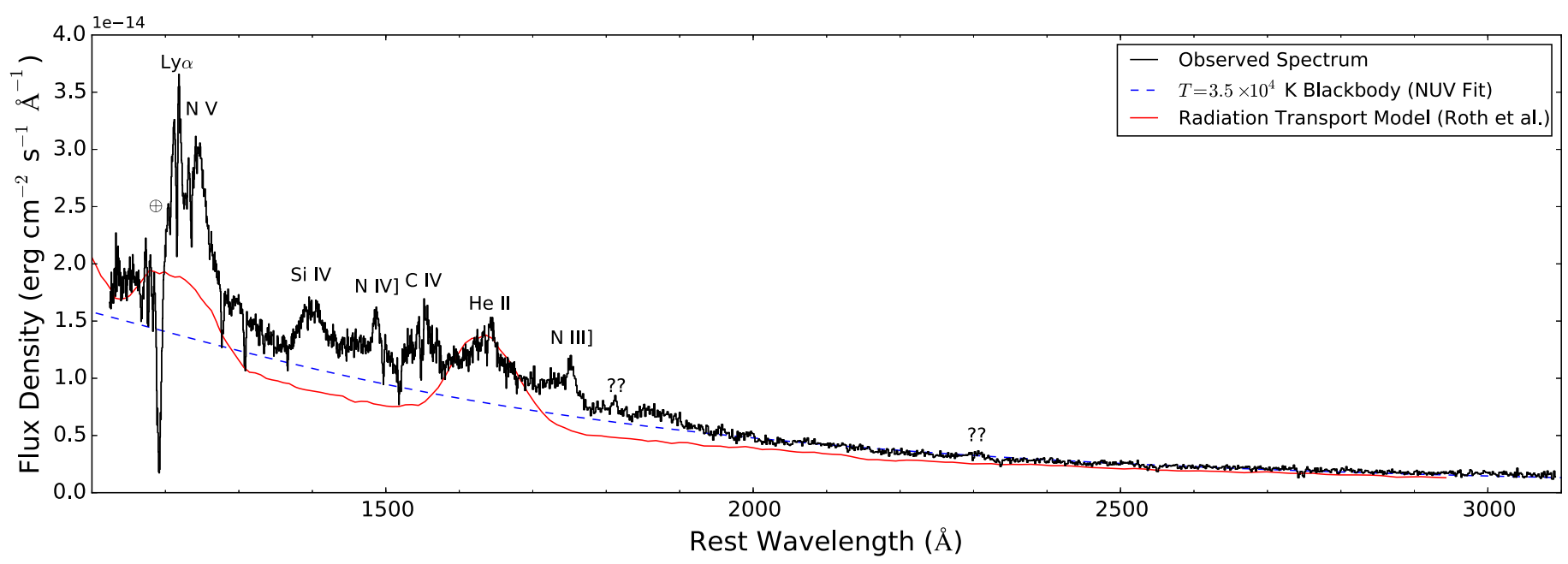

Figure 1. UV spectrum of the TDF ASASSN-14li. The broad emission features (where known) are indicated. Overplotted is a blackbody fit to the (largely featureless) NUV portion of the spectrum, with $T_{\mathrm{BB}}=(3.50 \pm 0.06) \times 10^{4} \mathrm{~K}$. Also shown is a radiative-transfer model from Roth et al. (2015). The portion of the spectrum affected by geocoronal airglow is indicated with a circled plus sign.

recently, a larger sample of optically discovered TDFs has revealed a wide range of $\mathrm{H}: \mathrm{He}$ line ratios (Arcavi et al. 2014), and still others appear to lack emission lines altogether (e.g., Cenko et al. 2012; Chornock et al. 2014).

Understanding the nature of this emission is critical for developing a theoretical framework to robustly infer SMBH properties (e.g., mass) from TDF observations. To this end, we have undertaken a campaign to obtain rest-frame ultraviolet (UV) spectra of TDF candidates with the Hubble Space Telescope (HST). By analogy with quasars, we anticipate that the strongest atomic lines will appear in the rest-frame UV. Similarly, the more-distant events discovered in the near future by, for example, the Large Synoptic Survey Telescope (LSST; Ivezic et al. 2008) will be observed at these rest-frame UV wavelengths. In this Letter we present the first spectrum obtained as part of this UV spectroscopy program, of the nearby TDF ASASSN-14li.

Throughout this work, we adopt a standard $\Lambda \mathrm{CDM}$ cosmology with parameters from Planck Collaboration et al. (2015): $H_{0}=67.8 \mathrm{~km} \mathrm{~s}^{-1} \mathrm{Mpc}^{-1}, \Omega_{m}=0.308$, and $\Omega_{\Lambda}=$ $1-\Omega_{m}$. All quoted uncertainties are $1 \sigma(68 \%)$ confidence intervals unless otherwise noted, and UTC times are used throughout.

\section{ASASSN-14li DISCOVERY AND OBSERVATIONS}

The All-Sky Automated Survey for Supernova (ASASSN; Shappee et al. 2014) first detected ASASSN-14li in $V$-band images obtained on 2014 November 11.65 in the nucleus of the galaxy PGC 043234 (Jose et al. 2014; Holoien et al. 2016). Preoutburst observations from the Sloan Digital Sky Survey (SDSS; Alam et al. 2015) indicate that the host galaxy is dominated by an old stellar population $\left(t_{\text {age }} \approx 11 \mathrm{Gyr}\right)$ with a stellar mass $\log _{10}\left(M_{*} / M_{\odot}\right) \approx 9.7$ and a redshift of $z=0.02058 \pm 0.00001$ (Conroy et al. 2009). At distance $d$ $\approx 90 \mathrm{Mpc}$, ASASSN-14li was exquisitely observed across the electromagnetic spectrum (Alexander et al. 2015; Miller et al. 2015; van Velzen et al. 2016; Holoien et al. 2016). We highlight the following previous results.

1. The broadband spectral energy distribution requires the presence of multiple emission components (van Velzen et al. 2016; Holoien et al. 2016). The observed X-ray emission is well fit by a blackbody with $T_{\mathrm{X}} \approx 5.8 \times 10^{5} \mathrm{~K}$ (Miller et al. 2015); however, extrapolating this model to optical wavelengths grossly underpredicts the observed flux. A second blackbody with $T_{\text {opt }} \approx 4 \times 10^{4} \mathrm{~K}$ can reasonably describe the optical emission, though the peak is largely unconstrained (van Velzen et al. 2016; Holoien et al. 2016).

2. Evidence for a high-velocity outflow $[\Delta v \approx(1.2-$ 3.9) $\left.\times 10^{4} \mathrm{~km} \mathrm{~s}^{-1}\right]$ and a low-velocity outflow $\left(\Delta v=-(100-400) \mathrm{km} \mathrm{s}^{-1}\right)$ was derived from nonthermal radio emission (Alexander et al. 2015; van Velzen et al. 2016) and high-resolution X-ray spectroscopy (Miller et al. 2015), respectively. Outflows are predicted to accompany accretion at super-Eddington rates (e.g., Ohsuga et al. 2005; Strubbe \& Quataert 2009), though typically only with large velocities.

3. The optical spectra of ASASSN-14li are dominated by a blue continuum and asymmetric, broad [FWHM $\approx(1-2) \times 10^{4} \mathrm{~km} \mathrm{~s}^{-1}$ ] emission lines of Balmer $\mathrm{H}$ and He II (Holoien et al. 2016).

We obtained UV spectra of ASASSN-14li with the Space Telescope Imaging Spectrograph (STIS; Program ID GO13853) on HST beginning at $08: 40$ on 2015 January 10 $(\Delta t=59.7$ day after initial detection). STIS was deployed with two different instrumental configurations, both utilizing the $52^{\prime \prime} \times 0$ ". 2 aperture: the G230L grating with the near-UV (NUV) MAMA detector, providing wavelength coverage of 1570-3180 $\AA$ at a resolution $(R \equiv \lambda / \Delta \lambda)$ of $\sim 700(1750 \mathrm{~s}$ exposure time), and the G140L grating with the far-UV (FUV) MAMA detector, providing wavelength coverage of $1150-1730 \AA$ at $R \approx 1700$ (2888 s exposure time).

We downloaded the processed frames from the HST archive and examined the resulting two-dimensional spectra. For both the FUV and NUV spectra, the trace from ASASSN-14li is well detected and (spatially) unresolved, so we make use of the standard pipeline product one-dimensional spectra for our analysis. After a signal-to-noise ratio $(\mathrm{S} / \mathrm{N})$-weighted combination of the FUV and NUV frames and dereddening for absorption in the Milky Way $(E(B-V)=0.022$ mag; Schlafly 
\& Finkbeiner 2011), the resulting UV spectrum of ASASSN14li is plotted in Figure 1. We caution that wavelengths near $1216 \AA$ are significantly affected by geocoronal airglow emission and will not be used for analysis here. Upon publication we will make the spectrum of ASASSN-14li available via WISeREP (Yaron \& Gal-Yam 2012).

\section{ANALYSIS}

\subsection{UV Continuum Emission}

To estimate the continuum level, we fit the portion of the spectrum with $\lambda \geqslant 1900 \AA$ to a blackbody function. ${ }^{22}$ The best-fit model, with $T_{\mathrm{BB}}=(3.50 \pm 0.06) \times 10^{4} \mathrm{~K}$, is overplotted in Figure 1. The FUV flux is, as expected, somewhat underpredicted by this model, but it seems to capture the continuum underlying the broad FUV emission features reasonably well. The total bolometric luminosity implied is $L_{\mathrm{BB}}=2.4 \times 10^{43} \mathrm{erg} \mathrm{s}^{-1}$, a factor of $\sim 2$ larger than the integrated emission in the observed bandpass $\left[L_{\mathrm{UV}}=(1.15 \pm\right.$ $0.06) \times 10^{43} \mathrm{erg} \mathrm{s}^{-1}$ ].

The derived UV continuum temperature at this epoch is consistent with previous estimates based on blackbody fits to broadband optical and near-UV photometry (van Velzen et al. 2015; Holoien et al. 2016). As noted by these authors, a single blackbody cannot simultaneous account for the luminous optical/UV and X-ray emission.

In Figure 1, we also overplot the results of a radiative-transfer simulation from Roth et al. (2015). This particular geometry consists of an outer envelope of $0.125 M_{\odot}$ of material extending out to $5 \times 10^{14} \mathrm{~cm}$ (a factor of $\sim 3$ larger than the radius inferred from our blackbody fits above), with a density profile of $\rho \propto r^{-2}$. The envelope is illuminated by an underlying continuum with a photospheric radius of $10^{14} \mathrm{~cm}$ and a bolometric luminosity of $10^{45} \mathrm{erg} \mathrm{s}^{-1}$. The resulting output spectrum is not a formal "fit" to the data, and furthermore only contains atomic species of $\mathrm{H}, \mathrm{He}$, and $\mathrm{O}$ at solar abundances. However, it is illustrative that the observed UV continuum slope is quite well reproduced with such a geometry.

\subsection{Absorption and Emission Features}

We fit emission and absorption features to a Gaussian model, allowing the central wavelength, line width, and equivalent width to vary as free parameters. The local continuum level was estimated from nearby wavelength bins. The results of this analysis are displayed in Table 1 . We identify three distinct classes of features: narrow absorption from the Milky Way, and narrow absorption and broad emission at or near the host redshift.

\subsubsection{Milky Way Absorption}

We detect a series of narrow (FWHM $\approx 500 \mathrm{~km} \mathrm{~s}^{-1}$ ) absorption features from standard metal transitions in the interstellar medium (ISM) at wavelengths near their rest values. Given their proximity to rest wavelengths and the lack of additional intervening material, we associate these features with an absorber in or near the Milky Way Galaxy. We calculate a weighted average redshift for the absorber of $z=$ $0.00064 \pm 0.00006$, or a velocity relative to the heliocentric reference frame of $v=190 \pm 20 \mathrm{~km} \mathrm{~s}^{-1}$. For the Galactic

\footnotetext{
$\overline{22}$ We assume the host-galaxy contribution at these wavelengths is minimal, as indicated by pre-outburst GALEX photometry (Miller et al. 2015).
}

coordinates of ASASSN-14li $\left(l=298^{\circ} .28, b=80^{\circ} .62\right)$, this results in a comparable velocity in the Local Standard of Rest. This velocity is indicative of a high-velocity cloud (HVC; Wakker \& van Woerden 1997).

\subsubsection{Absorption at Host Redshift}

While the absorption features from the Milky Way generally resemble those observed in the ISM of highredshift galaxies (e.g., Wolfe et al. 2005; Fynbo et al. 2009), the narrow absorption features observed from the host galaxy of ASASSN-14li are markedly different. With the notable exception of weak C II $\lambda 1335$, nearly all transitions from lowionization metal states are absent (Figure 2) ${ }^{23}$ Similarly, Ly $\alpha$ is extremely weak: assuming the gas is optically thin, we derive a lower limit to the column density of $\log N$ $\left(\mathrm{cm}^{-2}\right) \geqslant 14.2 \pm 0.2$. At first glance, the weak absorption is not entirely surprising, given the old stellar population observed in the host galaxy. But standard high-ionization absorption lines, such as $\mathrm{C}$ IV $\lambda \lambda 1548,1551$, S IV $\lambda \lambda 1394$, 1403 , and $\mathrm{N} v \lambda \lambda 1239,1243$ are well detected (Figure 3). We measure a weighted average for the absorber redshift of $z=0.02044 \pm 0.00006$, marginally below the value measured in the (quiescent) host.

\subsubsection{Emission at Host Redshift}

A number of broad emission features are also apparent, including $\mathrm{C}_{\text {IV }} \lambda \lambda 1548,1551, \mathrm{~S}_{\text {IV }} \lambda \lambda 1394,1403$, N v $\lambda \lambda 1239$, 1243, and $\operatorname{Ly} \alpha$. We further identify the feature at $\lambda_{\text {obs }}=1674 \AA$ as He II $\lambda 1640$, given the strong He II $\lambda 4686$ emission observed in the optical spectra of ASASSN-14li (Holoien et al. 2016). This line was predicted to appear in the UV spectra of TDFs by Strubbe \& Murray (2015), but with a P-Cygni profile not readily identified here. It also appears prominently in the radiative-transfer simulations of Roth et al. (2015) (Figure 1). ${ }^{24}$ This He II line may be blended with $\mathrm{O}$ III] $\lambda 1663$, but the low $\mathrm{S} / \mathrm{N}$ precludes firm conclusions.

We associate two additional features with $\mathrm{N}$ III] $\lambda 1750$ and $\mathrm{N}_{\text {Iv }} \lambda 1486$ (see also Kochanek 2015). We are unable to identify two remaining lines, both relatively weak, at $\lambda_{\text {host }} \approx$ $2303 \AA$ and $\lambda_{\text {host }} \approx 1812 \AA .{ }^{25}$ Equally of interest are common quasar absorption features that are entirely lacking from the UV spectrum of ASASSN-14li: $\mathrm{Mg}$ II $\lambda \lambda 2796,2804$ and C III] $\lambda 1909$ (Figure 4).

After manually excising narrow absorption features in the regions of interest, we fit the emission lines to a Gaussian model, as described above. The width of the emission features varies considerably, from FWHM $\approx 7700 \mathrm{~km} \mathrm{~s}^{-1}$ for $\mathrm{S}$ IV $\lambda \lambda 1394,1403$ to $1700 \mathrm{~km} \mathrm{~s}^{-1}$ for $\mathrm{N}$ iv] $\lambda 1486$ (corresponding to distances of $\sim 7-150$ au for virialized gas orbiting a $10^{6} M_{\odot}$ SMBH; van Velzen et al. 2016; Holoien et al. 2016). In fact, aside from one of the unidentified features, the two $\mathrm{N}$ transitions are significantly more narrow than the remainder of the lines. Even the largest velocities measured here are

\footnotetext{
${ }^{23}$ The feature at $\lambda_{\text {obs }}=2854 \AA$ could correspond either to $\mathrm{Mg}$ I $\lambda 2853$ from the Milky Way or $\mathrm{Mg}_{\text {III }} 2796$ from the TDF host. Given the lack of corresponding $\operatorname{Mg}{ }_{\text {II }} \lambda 2803$ at the host redshift, we associate this with the Milky Way absorber.

${ }^{24}$ We note that Roth et al. (2015) adopted a Gaussian line profile with Doppler velocity of $10^{4} \mathrm{~km} \mathrm{~s}^{-1}$ for their spectra; this profile and line width were not an output of the simulation.

25 The latter line appears in the composite N-rich QSO spectrum presented in Jiang et al. (2008) and may result from an Si II blend; see their Figure 4.
} 
Table 1

ASASSN-14li Absorption and Emission Features

\begin{tabular}{|c|c|c|c|c|c|c|}
\hline $\begin{array}{l}\lambda_{\mathrm{gbs}} \\
(\AA)\end{array}$ & Identification & $\begin{array}{l}\lambda_{0} \\
(\AA)\end{array}$ & $\begin{array}{l}W_{r}^{\mathrm{a}} \\
(\AA)\end{array}$ & $\begin{array}{l}\text { FWHM } \\
(\AA)\end{array}$ & $\begin{array}{c}\text { Line Flux } \\
\left(10^{-14} \mathrm{erg} \mathrm{cm}^{-2} \mathrm{~s}^{-1}\right)\end{array}$ & $z$ \\
\hline 2911.27 & $\mathrm{Mg}_{\mathrm{I}}$ & 2852.96 & $<1.0$ & 3.0 & $\ldots$ & 0.02044 \\
\hline 2860.83 & $\mathrm{Mg}_{\text {II }}$ & 2803.53 & $<0.65$ & 3.0 & $\ldots$ & 0.02044 \\
\hline 2856.3 & Mg II & 2798.75 & $>-2.8$ & 15.0 & $<0.8$ & 0.02058 \\
\hline $2854.35 \pm 0.42$ & $\mathrm{Mg}_{\mathrm{I}}$ & 2852.96 & $0.95 \pm 0.36$ & $4.0 \pm 1.4$ & $\ldots$ & $0.00049 \pm 0.00016$ \\
\hline $2805.70 \pm 0.24$ & Mg II & 2803.53 & $1.49 \pm 0.24$ & $3.8 \pm 0.6$ & $\ldots$ & $0.00077 \pm 0.00009$ \\
\hline $2799.04 \pm 0.21$ & Mg II & 2796.35 & $1.33 \pm 0.22$ & $3.2 \pm 0.5$ & $\cdots$ & $0.00096 \pm 0.00008$ \\
\hline 2653.32 & $\mathrm{Fe}$ II & 2600.17 & $<0.77$ & 3.0 & $\cdots$ & 0.02044 \\
\hline $2602.67 \pm 0.33$ & $\mathrm{Fe}$ II & 2600.17 & $0.79 \pm 0.20$ & $3.3 \pm 0.9$ & $\ldots$ & $0.00096 \pm 0.00013$ \\
\hline 2431.47 & Fe II & 2382.77 & $<0.61$ & 3.0 & $\ldots$ & 0.02044 \\
\hline 2392.13 & $\mathrm{Fe}$ II & 2344.21 & $<0.64$ & 3.0 & $\ldots$ & 0.02044 \\
\hline $2385.21 \pm 0.35$ & $\mathrm{Fe}$ II & 2382.77 & $0.69 \pm 0.24$ & $3.0 \pm 1.1$ & $\ldots$ & $0.00102 \pm 0.00015$ \\
\hline $2350.0 \pm 1.6$ & $? ? ?^{\mathrm{b}}$ & $\cdots$ & $-2.9 \pm 0.8$ & $20.7 \pm 6.0$ & $0.76 \pm 0.27$ & $\cdots$ \\
\hline $2346.78 \pm 0.20$ & $\mathrm{Fe}$ II & 2344.21 & $0.59 \pm 0.19$ & $2.0 \pm 0.6$ & $\cdots$ & $0.00110 \pm 0.00009$ \\
\hline 1948.0 & $\mathrm{C}_{\text {III }}$ & 1908.73 & $>-1.5$ & 15.0 & $<0.5$ & 0.02058 \\
\hline $1849.3 \pm 0.9$ & $? ? ?^{\mathrm{b}}$ & $\ldots$ & $-1.1 \pm 0.3$ & $5.9 \pm 2.6$ & $0.62 \pm 0.20$ & $\ldots$ \\
\hline $1788.0 \pm 0.7$ & $\mathrm{~N}$ III] & 1750.26 & $-2.7 \pm 0.6$ & $9.7 \pm 2.2$ & $2.1 \pm 0.6$ & $0.02156 \pm 0.00040$ \\
\hline 1704.94 & $\mathrm{Al}$ II & 1670.79 & $<0.33$ & 3.0 & $\ldots$ & 0.02044 \\
\hline $1673.7 \pm 0.6$ & He II & 1640.42 & $-4.5 \pm 0.6$ & $16.0 \pm 1.6$ & $4.6 \pm 0.5$ & $0.02028 \pm 0.00037$ \\
\hline $1671.58 \pm 0.22$ & $\mathrm{Al}$ II & 1670.79 & $0.40 \pm 0.14$ & $1.7 \pm 0.6$ & $\cdots$ & $0.00047 \pm 0.00013$ \\
\hline $1583.0 \pm 0.5$ & $\mathrm{C}$ IV & 1549.06 & $-4.8 \pm 0.7$ & $13.7 \pm 1.0$ & $5.0 \pm 0.8$ & $0.02191 \pm 0.00032$ \\
\hline $1582.60 \pm 0.14$ & $\mathrm{C}$ IV & 1550.77 & $0.52 \pm 0.12$ & $1.5 \pm 0.4$ & $\cdots$ & $0.02053 \pm 0.00009$ \\
\hline $1579.63 \pm 0.15$ & $\mathrm{C}_{\mathrm{IV}}$ & 1548.20 & $1.24 \pm 0.22$ & $3.0 \pm 0.5$ & $\ldots$ & $0.02030 \pm 0.00009$ \\
\hline 1557.92 & Si II & 1526.71 & $<0.25$ & 3.0 & $\cdots$ & 0.02044 \\
\hline $1551.93 \pm 0.23$ & $\mathrm{C}$ IV & 1550.77 & $0.48 \pm 0.17$ & $1.6 \pm 0.6$ & $\cdots$ & $0.00075 \pm 0.00015$ \\
\hline $1549.16 \pm 0.21$ & $\mathrm{C}_{\text {IV }}$ & 1548.20 & $1.07 \pm 0.22$ & $2.8 \pm 0.6$ & $\ldots$ & $0.00062 \pm 0.00014$ \\
\hline $1527.37 \pm 0.14$ & Si II & 1526.71 & $0.90 \pm 0.16$ & $2.8 \pm 0.5$ & $\cdots$ & $0.00043 \pm 0.00009$ \\
\hline $1517.3 \pm 0.3$ & $\mathrm{~N}$ IV] & 1486.50 & $-2.3 \pm 0.4$ & $8.4 \pm 1.1$ & $2.4 \pm 0.4$ & $0.02072 \pm 0.00020$ \\
\hline 1431.44 & Si IV & 1402.77 & $<0.21$ & 3.0 & $\ldots$ & 0.02044 \\
\hline $1426.7 \pm 0.5$ & Si IV & 1396.76 & $-10.9 \pm 1.1$ & $35.7 \pm 2.0$ & $11.5 \pm 1.0$ & $0.02144 \pm 0.00036$ \\
\hline $1422.48 \pm 0.17$ & Si IV & 1393.76 & $0.21 \pm 0.07$ & $1.3 \pm 0.5$ & $\ldots$ & $0.02061 \pm 0.00012$ \\
\hline $1394.53 \pm 0.13$ & Si IV & 1393.76 & $0.20 \pm 0.06$ & $0.8 \pm 0.3$ & $\ldots$ & $0.00055 \pm 0.00009$ \\
\hline $1361.66 \pm 0.19$ & $\mathrm{C}_{\text {II }}$ & 1334.53 & $0.35 \pm 0.11$ & $2.5 \pm 0.6$ & $\cdots$ & $0.02033 \pm 0.00014$ \\
\hline $1335.27 \pm 0.08$ & $\mathrm{C}_{\text {II }}$ & 1334.53 & $1.26 \pm 0.14$ & $3.4 \pm 0.3$ & $\ldots$ & $0.00055 \pm 0.00006$ \\
\hline 1331.03 & Si II & 1304.37 & $<0.11$ & 3.0 & $\ldots$ & 0.02044 \\
\hline 1328.79 & $\mathrm{O}_{\mathrm{I}}$ & 1302.17 & $<0.12$ & 3.0 & $\ldots$ & 0.02044 \\
\hline $1305.22 \pm 0.14$ & Si II & 1304.37 & $0.39 \pm 0.07$ & $1.4 \pm 0.3$ & $\cdots$ & $0.00065 \pm 0.00011$ \\
\hline $1302.98 \pm 0.10$ & $\mathrm{O}_{\mathrm{I}}$ & 1302.17 & $0.52 \pm 0.07$ & $1.6 \pm 0.3$ & $\ldots$ & $0.00062 \pm 0.00008$ \\
\hline 1286.18 & $\mathrm{Si}$ II & 1260.42 & $<0.35$ & 3.0 & $\cdots$ & 0.02044 \\
\hline $1268.60 \pm 0.22$ & $\mathrm{~N} v$ & 1242.80 & $0.30 \pm 0.13$ & $0.7 \pm 0.5$ & $\ldots$ & $0.02076 \pm 0.00018$ \\
\hline $1264.40 \pm 0.25$ & $\mathrm{~N} v$ & 1238.82 & $0.52 \pm 0.14$ & $1.2 \pm 0.5$ & $\ldots$ & $0.02065 \pm 0.00020$ \\
\hline $1260.74 \pm 0.09$ & Si II & 1260.42 & $0.59 \pm 0.08$ & $2.3 \pm 0.3$ & $\cdots$ & $0.00025 \pm 0.00007$ \\
\hline $1240.49 \pm 0.04$ & $\operatorname{Ly} \alpha$ & 1215.67 & $0.83 \pm 0.04$ & $2.0 \pm 0.1$ & $\ldots$ & $0.02042 \pm 0.00003$ \\
\hline
\end{tabular}

Notes.

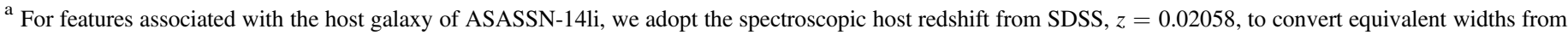

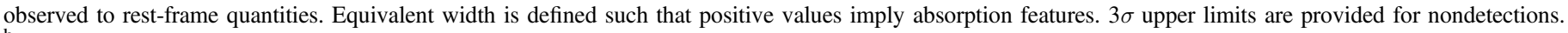

${ }^{\mathrm{b}}$ We are unable to identify the atomic transition responsible for this observed feature.

substantially smaller than those measured from optical lines [FWHM $\approx(1-2) \times 10^{4} \mathrm{~km} \mathrm{~s}^{-1}$; Holoien et al. 2016].

For features where both narrow absorption and broad emission are detected, the absorption features are blueshifted: $\Delta v=-450 \pm 100 \mathrm{~km} \mathrm{~s}^{-1}$ for $\mathrm{C}$ Iv $\lambda \lambda 1548, \quad 1551$, and $\Delta v=-250 \pm 110 \mathrm{~km} \mathrm{~s}^{-1}$ for S IV $\lambda \lambda 1394,1403$.

Finally, we note that we did not attempt to fit either the broad Ly $\alpha$ or $\mathrm{N} v \lambda \lambda 1239,1243$ host emission features, owing to both their clear blending and the large number of narrow absorption features at these wavelengths. The absorption feature blueward of $\operatorname{Ly} \alpha$ is from $\mathrm{H}_{\mathrm{I}}$ gas in the HVC; none of the lines shows evidence for a P-Cygni profile.

\section{DISCUSSION}

As a starting place for discussion, the most natural point of comparison is with quasars (QSOs), given that both are powered by accretion onto a SMBH. In Figure 4 we overplot the composite QSO spectrum from SDSS (Vanden Berk et al. 2001). Even compared with a QSO, the continuum emission from ASASSN-14li is significantly bluer. Together with the luminous, thermal X-ray emission, the nature of the continuum in ASASSN-14li appears to be significantly different from that observed in typical QSOs.

In terms of line features, our first task is to understand the source of the emitting and absorbing material. The lack of 

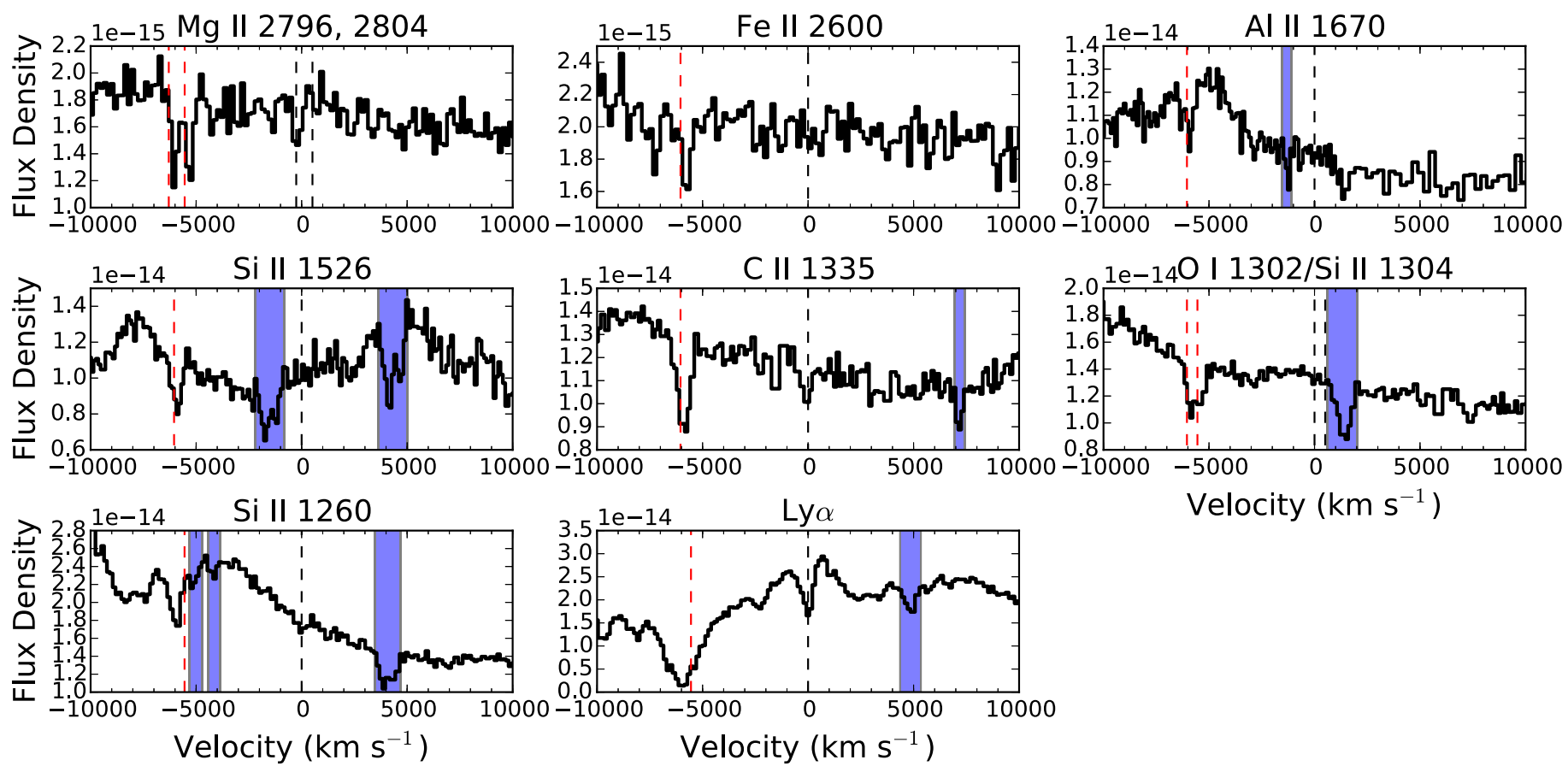

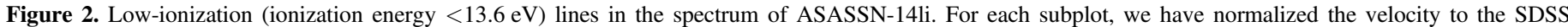

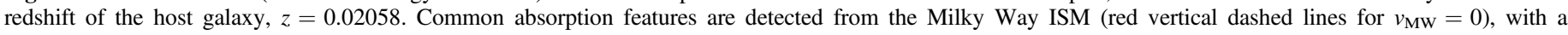
significant velocity offset. Regions contaminated by features from other transitions are shaded blue.
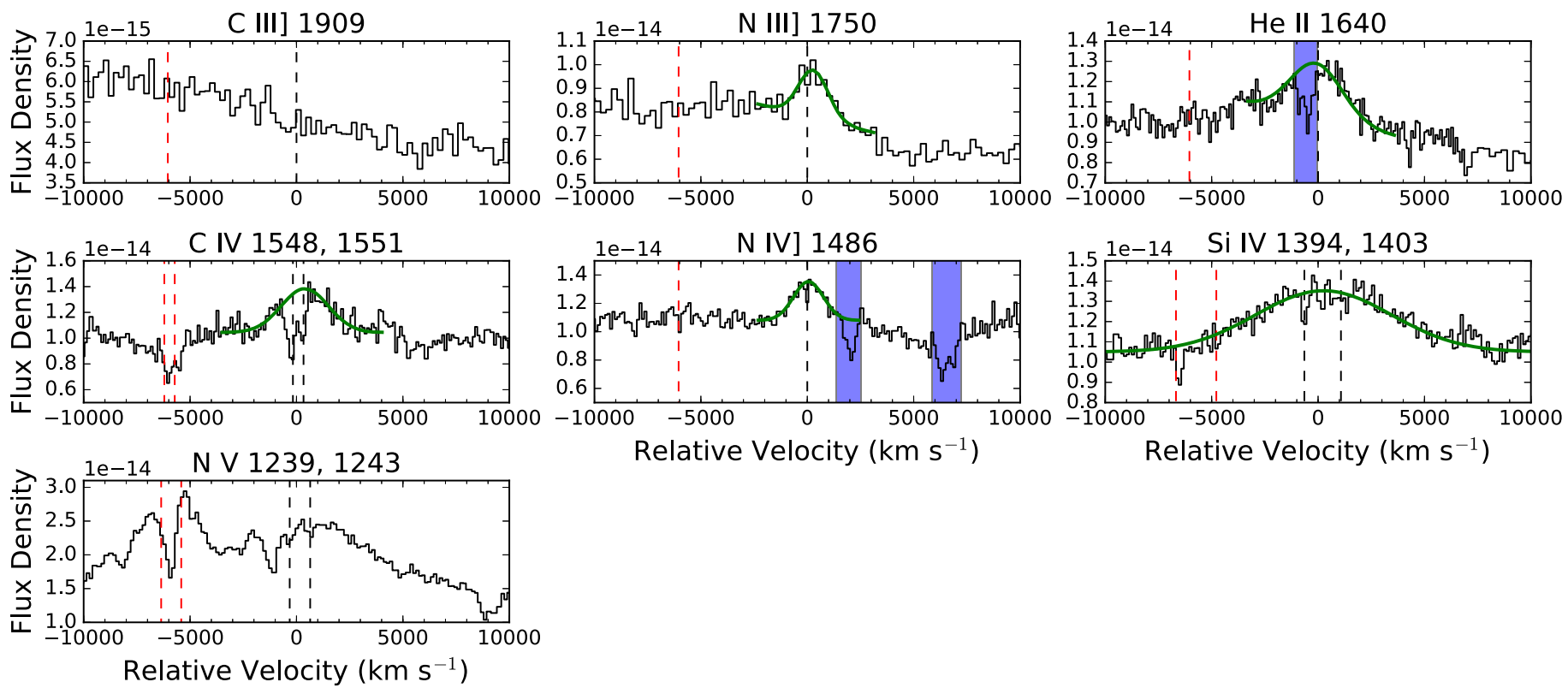

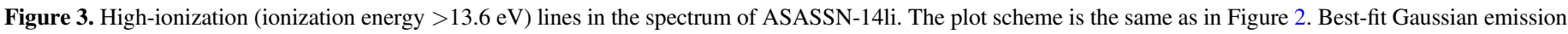
models are plotted in green.

common low-ionization absorption features, together with the old host stellar population, indicate that the absorber is unlikely to arise in the cold host ISM. Given that nearly every $L_{*}$ galaxy exhibits a circumgalactic medium (GGM) consisting of strong Ly $\alpha$ absorption and (frequently) low-ionization absorption (Prochaska et al. 2011; Thom et al. 2012), there is a reasonable probability that gas in the halo of the host galaxy contributes to the observed absorption. However, the detection of $\mathrm{N} v \lambda \lambda 1239,1243$ is difficult to account for in such a CGM environment (J. K. Werk et al. 2016, in preparation).
The host galaxy does show evidence for a pre-outburst AGN, and so the absorber could result from pre-existing gas in (or possibly expelled from; van Velzen et al. 2016) the nuclear region. Alternatively, the absorber may result from the (bound) debris of the disrupted star. We argue this is the simplest explanation, as the same material responsible for the X-ray outflow (Miller et al. 2015) could also produce UV absorption (e.g., Crenshaw et al. 1999; Kriss 2006). The comparable blueshift (with respect to the broad emission lines) observed in the UV and X-rays further supports this conclusion. 


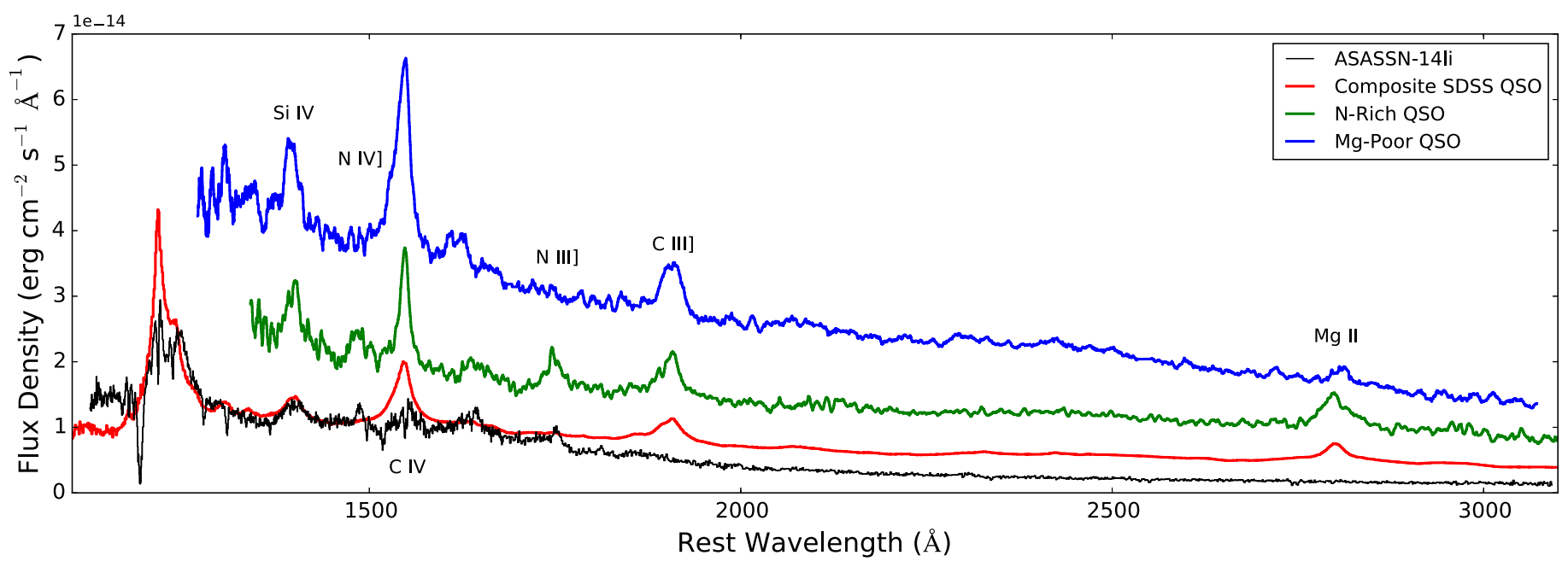

Figure 4. The UV spectrum of ASASSN-14li, compared with the composite QSO spectrum from SDSS (red; Vanden Berk et al. 2001), an "N-rich" QSO (SDSS J125414.27+024117.5), and an "Mg-poor" QSO (SDSS J003922.44+005951.7).

The emission lines present in ASASSN-14li are to first order analogous to a BLR in a QSO: clearly there is fast-moving $\left(\gtrsim 5 \times 10^{3} \mathrm{~km} \mathrm{~s}^{-1}\right)$ material photoionized (or collisionally excited) by the blue continuum. However, closer examination reveals an extremely unusual abundance pattern. Strong $\left.\mathrm{N}_{\text {IV }}\right] \lambda 1486$ and $\left.\mathrm{N}_{\mathrm{III}}\right] \lambda 1750$ are extremely rare in QSOs, with only $\sim 1 \%$ of SDSS systems exhibiting such features (Jiang et al. 2008). The lack of $\mathrm{C}_{\text {III }} \lambda 1909$ and $\mathrm{Mg}$ II $\lambda \lambda 2796,2804$ emission is particularly striking.

We have searched through the SDSS QSO database to identify other examples of systems that either (a) exhibit strong $\left.\mathrm{N}_{\text {IV }}\right] \lambda 1486$ and $\mathrm{N}$ III] $\lambda 1750$ emission, and/or (b) display large C Iv $\lambda \lambda 1548,1551$ equivalent widths, but weak or nonexistent Mg II $\lambda \lambda 2796,2804$ emission. As noted by Kochanek (2015), the former group has been previously identified as the "N-rich" QSOs (Osmer 1980; Bentz \& Osmer 2004; Bentz et al. 2004). Kochanek (2015) argues that these N-rich QSOs are in fact the tidal disruption of a $\gtrsim 1 M_{\odot}$ star, for which the high $\mathrm{N}$ abundance results from significant $\mathrm{CNO}$ processing in the stellar core (predisruption).

While the similarity between N-rich QSOs and ASASSN14li is indeed intriguing, we note several important caveats. First, we examined all the $\mathrm{N}$-rich systems identified by Jiang et al. (2008), and every one (in the appropriate redshift range) exhibited detectable $\mathrm{C}_{\text {III }} \lambda 1909$ and $\mathrm{Mg}$ II $\lambda \lambda 2796$, 2804 emission lines. $\mathrm{Mg}$ should be unaffected by the CNO cycle. The absence of $\mathrm{Mg}$ II could be the result of photoionization from the extremely hot continuum: given that the ionization energy of $\mathrm{Mg}$ III is $80.1 \mathrm{eV}$ (comparable to the ionization energy of $\mathrm{N}_{\text {IV }}$ of $77.5 \mathrm{eV}$ ), there are clearly many photons capable of further electron stripping. In this case, the absence of $\mathrm{Mg}$ II may be transient in nature, as the underlying continuum temperature must cool eventually.

In addition, only a small fraction $(\lesssim 10 \%)$ of the N-rich QSOs exhibit narrow, blueshifted absorption lines as were observed for ASASSN-14li. Again, however, if related to accretion at super-Eddington rates, these absorption features could also be transient in nature. Future spectra of ASASSN14li, when the accretion rate has dropped and the continuum cooled, are a critical test of this explanation.

We also plot in Figure 4 an example QSO spectrum with well-detected C Iv $\lambda \lambda 1548$, 1551, but weak or nonexistent
$\operatorname{Mg}$ II $\lambda \lambda 2796,2804$ emission. Of the 78,223 QSOs in the catalog of Shen et al. (2011) with well-detected C Iv $\lambda \lambda 1548$, 1551 and $z<2$, only $269(0.3 \%)$ lack detectable Mg II $\lambda \lambda 2796$, $2804\left(W_{r}<3 \sigma\right)$. We visually inspected these 269 "Mg-poor" QSOs, and in nearly all cases the lack of $\mathrm{Mg}$ II $\lambda \lambda 2796,2804$ is due to decreased sensitivity at the relevant (observed) wavelength. All these sources have well-detected C III] $\lambda 1909$ lines.

Detailed photoionization modeling may help to shed further light on the nature of the emitting gas. For example, the lack of $\mathrm{C}_{\text {III }} \lambda 1909$ could be explained if the gas were above the critical density of $n_{\text {crit }}=10^{9.5} \mathrm{~cm}^{-3}$ (Osterbrock 1989); constraints from the X-ray spectrum place a lower limit of $n \gtrsim$ $2 \times 10^{9} \mathrm{~cm}^{-3}$. Similarly, if the narrow width of the (semiforbidden) $\mathrm{N}$ lines were caused by collisional de-excitation (and not, say, distance from the source), this may also constrain the density of the emitting gas. However, we caution that assumptions underlying standard AGN photoionization tools (e.g., CLOUDY; Ferland et al. 2013) may not hold for TDFs (Roth et al. 2015).

To the extent that ASASSN-14li is representative of the broader TDF population, the UV spectrum is extremely promising for the detection of high-redshift events by future wide-field optical surveys. With the blue continuum (and no evidence for dust), the resulting negative K-correction will greatly enhance detectability: ASASSN-14li, for example, would be easily detectable by LSST out to $z \approx 1$ $\left(m_{g} \approx 25.0 \mathrm{mag}\right.$ at $\left.d_{\mathrm{L}}=6.8 \mathrm{Gpc}\right)$. Furthermore, the extremely blue continuum should clearly distinguish TDFs from other classes of transients (van Velzen et al. 2011).

However, based on this result, it is clear that we have yet to reach a complete picture of the process by which the emission is generated following the tidal disruption process. The simplest analytic models (e.g., Ulmer 1999; Strubbe \& Quataert 2009; Lodato \& Rossi 2011), which assume rapid circularization of the bound debris, may miss fundamental physics governing the observed signal (Guillochon \& RamirezRuiz 2015; Piran et al. 2015; Shiokawa et al. 2015). As is clear from our spectrum of ASASSN-14li, the line formation is a complex process, and QSOs are an imperfect analog. Without improved understanding of how (and where) the emission is 
generated, it will be quite challenging to utilize TDFs as probes of distant SMBHs.

Finally, the path forward (observationally) is quite clear. Time-resolved spectra should solve many of the puzzles presented here. A reverberation mapping campaign (e.g., Peterson 1993) would be an incredibly powerful discriminant for future events. Furthermore, additional nearby examples are necessary to determine if ASASSN-14li is indeed a fair representation of the broader TDF population, or if something unique about ASASSN-14li (nature of the disrupted star, orbit, geometry, etc.) gives rise to both luminous X-ray and optical/ UV emission.

We thank R. Chornock, M. Eracleous, P. Hall, and C. Kochanek for valuable discussions, and the $H S T$ staff for the prompt scheduling of these ToO observations. S.B.C. acknowledges the Aspen Center for Physics and NSF Grant \#1066293 for hospitality. AVF's research was funded by NSF grant AST-1211916, the TABASGO Foundation, and the Christopher R. Redlich Fund.

Based on observations made with the NASA/ESA Hubble Space Telescope, obtained from the Data Archive at the Space Telescope Science Institute, which is operated by the Association of Universities for Research in Astronomy, Inc., under NASA contract NAS 5-26555.

Facility: HST (STIS).

\section{REFERENCES}

Alam, S., Albareti, F. D., Allende Prieto, C., et al. 2015, ApJS, 219, 12 Alexander, K. D., Berger, E., Guillochon, J., Zauderer, B. A., \& Williams, P. K. G. 2015, arXiv:1510.01226

Arcavi, I., Gal-Yam, A., Sullivan, M., et al. 2014, ApJ, 793, 38

Bentz, M. C., Hall, P. B., \& Osmer, P. S. 2004, AJ, 128, 561

Bentz, M. C., \& Osmer, P. S. 2004, AJ, 127, 576

Bogdanović, T., Cheng, R. M., \& Amaro-Seoane, P. 2014, ApJ, 788, 99

Cenko, S. B., Krimm, H. A., Horesh, A., et al. 2012, MNRAS, 420, 2684

Chornock, R., Berger, E., Gezari, S., et al. 2014, ApJ, 780, 44

Conroy, C., Gunn, J. E., \& White, M. 2009, ApJ, 699, 486

Crenshaw, D. M., Kraemer, S. B., Boggess, A., et al. 1999, ApJ, 516, 750

De Colle, F., Guillochon, J., Naiman, J., \& Ramirez-Ruiz, E. 2012, ApJ, 760, 103

Ferland, G. J., Porter, R. L., van Hoof, P. A. M., et al. 2013, RMxAA, 49, 137
Fynbo, J. P. U., Jakobsson, P., Prochaska, J. X., et al. 2009, ApJS, 185,526

Gaskell, C. M., \& Rojas Lobos, P. A. 2014, MNRAS, 438, L36

Gezari, S., Chornock, R., Rest, A., et al. 2012, Natur, 485, 217

Guillochon, J., Manukian, H., \& Ramirez-Ruiz, E. 2014, ApJ, 783, 23

Guillochon, J., \& Ramirez-Ruiz, E. 2015, ApJ, 809, 166

Hills, J. G. 1975, Natur, 254, 295

Holoien, T. W.-S., Kochanek, C. S., Prieto, J. L., et al. 2016, MNRAS, 455, 2918

Ivezic, Z., Tyson, J. A., Abel, B., et al. 2008, arXiv:0805.2366

Jiang, L., Fan, X., \& Vestergaard, M. 2008, ApJ, 679, 962

Jose, J., Guo, Z., Long, F., et al. 2014, ATel, 6777

Kochanek, C. S. 2015, MNRAS, submitted (arXiv:1512.03065)

Kriss, G. A. 2006, in ASP Conf. Ser. 348, Astrophysics in the Far Ultraviolet Five Years of Discovery with FUSE, ed. G. Sonneborn, H. W. Moos, \& B.-G. Andersson (San Francisco, CA: ASP), 499

Lodato, G., \& Rossi, E. M. 2011, MNRAS, 410, 359

Miller, J. M., Kaastra, J. S., Miller, M. C., et al. 2015, Natur, 526, 542

Ohsuga, K., Mori, M., Nakamoto, T., \& Mineshige, S. 2005, ApJ, 628, 368

Osmer, P. S. 1980, ApJ, 237, 666

Osterbrock, D. E. 1989, Astrophysics of Gaseous Nebulae and Active Galactic Nuclei (Mill Valley, CA: Univ. Science Books)

Peterson, B. M. 1993, PASP, 105, 247

Phinney, E. S. 1989, in IAU Symp. 136, The Center of the Galaxy, ed. M. Morris (Dordrecht: Kluwer Academic), 543

Piran, T., Svirski, G., Krolik, J., Cheng, R. M., \& Shiokawa, H. 2015, ApJ, 806, 164

Planck Collaboration, Ade, P. A. R., Aghanim, M., et al. 2015, arXiv: 1502.01589

Prochaska, J. X., Weiner, B., Chen, H.-W., Mulchaey, J., \& Cooksey, K. 2011, ApJ, 740, 91

Rees, M. J. 1988, Natur, 333, 523

Roth, N., Kasen, D., Guillochon, J., \& Ramirez-Ruiz, E. 2015 , arXiv: 1510.08454

Schlafly, E. F., \& Finkbeiner, D. P. 2011, ApJ, 737, 103

Shappee, B. J., Prieto, J. L., Grupe, D., et al. 2014, ApJ, 788, 48

Shen, Y., Richards, G. T., Strauss, M. A., et al. 2011, ApJS, 194, 45

Shiokawa, H., Krolik, J. H., Cheng, R. M., Piran, T., \& Noble, S. C. 2015, ApJ, 804,85

Strubbe, L. E., \& Murray, N. 2015, MNRAS, 454, 2321

Strubbe, L. E., \& Quataert, E. 2009, MNRAS, 400, 2070

Thom, C., Tumlinson, J., Werk, J. K., et al. 2012, ApJL, 758, L41

Ulmer, A. 1999, ApJ, 514, 180

Vanden Berk, D. E., Richards, G T., Bauer, A., et al. 2001, AJ, 122, 549

van Velzen, S., Anderson, G. E., Stone, N. C., et al. 2016, Sci, 351, 62

van Velzen, S., Farrar, G. R., Gezari, S., et al. 2011, ApJ, 741, 73

Wakker, B. P., \& van Woerden, H. 1997, ARA\&A, 35, 217

Wolfe, A. M., Gawiser, E., \& Prochaska, J. X. 2005, ARA\&A, 43, 861

Yaron, O., \& Gal-Yam, A. 2012, PASP, 124, 668 